\title{
$\begin{array}{ll}\text { Research Square } & \text { Preprints are preliminary reports that have not undergone peer review. }\end{array}$ or referenced by the media as validated information. \\ Genome-wide investigation of heat shock transcription factor family in wheat (Triticum aestivum L.)
}

Jiali Ye

Northwest Agriculture and Forestry University https://orcid.org/0000-0003-2294-9084

\section{Xuetong Yang}

Northwest A\&F University

Sha Li

Northwest Agriculture and Forestry University

Wei Li

Northwest A\&F University

Qi Liu

Northwest Agriculture and Forestry University

Lingli Zhang

Northwest A\&F University

Xiyue Song ( $\nabla$ songxiyue@126.com )

\section{Research article}

Keywords: Evolution, Genome-wide, Heat Shock Transcription Factor, Male Sterility, Thermosensitivity,Wheat

Posted Date: May 2nd, 2019

DOl: https://doi.org/10.21203/rs.2.9414/v1

License: (c) (i) This work is licensed under a Creative Commons Attribution 4.0 International License. Read Full License 


\section{Abstract}

Background: Heat shock transcription factors (HSFs) play crucial roles in resisting heat stress and regulating plant development. Investigating the HSF family is essential for understanding the fertility conversion mechanism in thermo-sensitive male sterile wheat. Previous studies have investigated the HSF family in wheat but it is necessary to conduct more in-depth and systematic analyses based on the newly published reference genome. Results: In the present study, 61 wheat Hsf (TaHsf) genes were identified using two main strategies and renamed based on their physical locations on chromosomes. According to the gene structure and phylogenetic analyses, the 61 TaHsf genes were classified into three categories and eleven subclasses. The genes were unequally distributed on 21 chromosomes, including two pairs of tandem duplication genes and 52 TaHsf segmental duplication genes. According to the ciselements identified, most of the TaHsfs can be activated by Ca++ and MYB, and they respond to drought, light, copper, and other stresses as well as heat shock. RNA-seq analysis indicated that the A2 class TaHsf genes exhibited persistently upregulated expression levels in the leaves/shoots, roots (except in the vegetative growth and reproductive growth stages), spikes, and grains in wheat under normal conditions. The $\mathrm{A}$ and $\mathrm{B}$ class TaHsf genes were positively regulated during the resistance to heat, whereas the $\mathrm{C}$ class genes were involved in drought regulation in wheat. Only the A and B class TaHsf genes were upregulated under fertile conditions in thermo-sensitive male sterile wheat. Conclusion: In this study, 61 wheat Hsf genes were identified based on the complete wheat reference genome. This comprehensive analysis provides novel insights into the TaHsf genes, including their diverse functions and involvement in metabolic pathways.

\section{Background}

The heat shock response is common in plants where a series of stress responses are generated under external stresses, especially during heat stress [1]. Under heat stress, heat shock elements (HSEs) present in the promoter regions upstream of the heat shock protein (HSP) genes are recognized by the activated heat shock transcription factors (HSFs), and they induce the transcription of Hsp genes as molecular chaperones to help refold, assemble, distribute, and degrade related proteins, as well as repairing damaged proteins and maintaining cell survival [2-4]. In particular, regulation of the activity of HSFs is the key mechanism responsible for the transcription and expression of Hsp genes.

A typical HSF has four components comprising the N-terminal DNA-binding domain (DBD), oligomeric domain (OD), nuclear localization signal (NLS), and nuclear export signal (NES). Some HSFs also have a C-terminal activation domain (CTAD) [5]. DBDs are located at the N-terminus of HSFs and they are highly conserved regions with three helical structures $(\mathrm{H} 1, \mathrm{H} 2$, and $\mathrm{H} 3)$ and four inverted parallel $\beta$-sheets $(\beta 1$, $\beta 2, \beta 3$, and $\beta 4$ ) [6]. The helix-turn-helix (H2-T-H3) structure at the hydrophobic centre of the DBD allows the precise localization and recognition of HSE sequences (5-AGAAnnTTCT-3) [7]. The OD region is also located at the N-terminus of HSFs and it is linked to the DBD region by a basic amino acid sequence measuring 15 to 80 amino acids (aa) in length. The OD region comprises two hydrophobic heptad repeat regions: A and B (HR-A/B). HR-A contains 5-6 sets of hydrophobic heptapeptide repeats, whereas HR-B 
has two overlapping heptapeptide repeats, which form a helical coiled-coil structure in space [8]. According to the characteristics of the HR-A/B region, plant HSFs can be classified into class $A$, class $B$, and class $C$. Class A and class $C$ HSFs have 21 and 7 aa inserts between regions HR-A and HR-B, respectively, whereas class $B$ HSFs are relatively simple with no amino acid insertions [9]. The synergy between the NLS and NES maintains the cell balance, thereby allowing plant HSFs to be freely distributed in the cytoplasm and nucleus [10]. The CTAD is characterized by aromatic and large hydrophobic amino acids, and acidic amino acid (AHA) motifs specifically activate the HSFs in class A. By contrast, the B and $\mathrm{C}$ class HSFs have no activation functions due to the lack of an AHA domain [5].

The first HSF gene in plants was cloned in tomato (Solanum lycopersicum L.) [11] and they have now been characterized in Arabidopsis, rice [12], maize (Zea mays L.) [13], soybean (Glycine max L.) [14], chickpea [15], and sorghum [16], as well as in vegetables including Chinese cabbage [17] and pepper [18], and fruits such as apple [19] and pear [20]. In addition to those Hsfs who involved in the regulation of heat-resistance mechanisms were researched in Arabidopsis [21, 22], many Hsfs have been found to be participate in other response in various plants in recent years. The overexpression of AtHsfA2 improves the heat tolerance of Arabidopsis but it also enhances the salt tolerance [23], hypoxia resistance [24], and resistance to oxidative stress [25]. Under high temperature stress, the overexpression of LeHsfA2 increases the fruit set rate in tomato and enhances fruit development, probably because LeHsfA2 activates the protective mechanism for tomato pollen [26]. Shim et al. found that the overexpression of HsfA4a in rice increased the tolerance of cadmium [27]. The sunflower HaHsfA9 is involved in the development of the embryo and seed maturation. The overexpression of HaHsfA9 alone or HaHsfA9 together with HaDreb2 can also promote the synthesis and accumulation of HSPs in tobacco seeds and increase the seed life [28]. The class B and C HSFs lack transcriptional activities but many studies in recent years have shown that class $\mathrm{B}$ and class $\mathrm{C}$ Hsfs are essential for the regulation of plant growth and development, metabolism, and stress responses. For example, the heat-induced tomato LeHsfB1 is a coactivator of a class A Hsfs (e.g., HsfA1a) and it has an auxiliary activation function by enhancing the expression of target genes [29]. Little studies have shown that class C Hsfs can be induced by a variety of stresses [17].

Higher temperatures from wheat emergence until the tillering stage can decrease the number of tillers, while exposure to high temperatures from the jointing to flowering stages leads to a decreased numbers of panicles per ear and spikelets per panicle, and a lower grain weight [30]. Wheat is a major food crop so improving its heat resistance and ensuring food production is important for human society. Members of the HSF family in wheat have been identified based on the old reference genome [31], but the lack of sufficient information hinders in-depth studies of the stress resistance mechanisms related to TaHsfs. Indeed, only three members of the wheat HSF family have been functionally analyzed, i.e., TaHsfA4a, TaHsfB2, and TaHsfA6e[27, 32, 33]. Therefore, a more comprehensive and systematic analysis is required for TaHsfs based on the newly published wheat reference genome to provide a secure basis for subsequent research. 
The fertility of thermo-sensitive male sterile wheat is controlled by the temperature and it has been applied in wheat hybridization to increase the wheat yield. Understanding the fertility conversion mechanism is important for accelerating the rate of production improvements. In our previous studies, we showed that the expression levels of $H s f s$ and $H s p s$ differ significantly under different fertility conditions (different temperatures). In order to further explore their roles in the fertility conversion mechanism and the HSF family members that might be important for their roles, we used the newly published reference genome to identify the HSF family members in wheat. We analyzed their evolutionary relationships, gene structure, and expression patterns, and verified their preferential expression in thermo-sensitive male sterile wheat.

\section{Materials And Methods}

Genome-wide identification of the TaHsf family members in Triticum aestivum L.

The latest wheat reference genome and the hidden HMM profile of the HSF family in the wheat genome database IWGSC (IWGSC RefSeq annotation v1.1, https://wheat-urgi.versailles.inra.fr/SeqRepository/Annotations) [55] and Pfam database (http://pfam.xfam.org/family/PF00447) were downloaded [56]. The HSF HMM profile was searched in the wheat genome with the HMMER search program and reliable results were screened based on an E-value less than or equal to $1 \mathrm{e}-20$. The results obtained were used to construct a wheat-specific HSF HMM profile, before searching the wheat reference genome again with an E-value threshold of $1 \mathrm{e}-3$. In order to avoid missing other HSF members, the HSF protein sequences for rice and Arabidopsis were downloaded from phytozome (https://phytozome.jgi.doe.gov/pz/portal.html) and used as queries to BLAST the wheat reference genome, where the E-value threshold was $1 \mathrm{e}-10$. The results obtained were identified as wheat candidate genes and verified using CD-search (https://www.ncbi.nlm.nih.gov/Structure/bwrpsb/bwrpsb.cgi) [57], SMART (http://smart.embl.de/), and pfam (http://pfam.xfam.org/) [58]. Coiled-coil structures were detected using the MARCOIL program (https://bcf.isb-sib.ch/webmarcoil/webmarcoilC1.html) [59]. Protein sequences that lacked the DBD domain or coiled-coil structure were removed. According to the positions of the genes on the chromosomes, the non-redundant Hsfs were designated as TaHsf-01 to TaHsf-61. All high confidence level HSF protein sequences were submitted to Expy (https://www.expasy.org/structural_bioinformatics) to calculate the number of amino acids, molecular weight, and theoretical isoelectric point. NetNES (http://www.cbs.dtu.dk/services/NetNES/) and cNLS Mapper (http://nls-mapper.iab.keio.ac.jp/cgi-bin/NLS_Mapper_form.cgi\#opennewwindow) were used to search for the NESs and NLSs in the TaHsfs [60,61]. The gene annotation information for the TaHsf family members was submitted to the online site MapGene2 Chrom web V2 (http://mg2c.iask.in/mg2c_v2.0/) to map their chromosomal locations.

Multiple sequence alignment, phylogenetic analysis, and classification of TaHsfs

The full-length amino acid sequences of the HSFs in rice and Arabidopsis were used for evolutionary analysis with the full-length amino acid sequences of the TaHsfs. First, all of the sequences were 
imported into MAGE7 for sequence comparison using the default parameters and the phylogenetic tree was then constructed based on the conserved regions in the alignment results with the maximum likelihood method, where the settings comprised the Poisson distribution mode and bootstrap tests were conducted with 1000 replicates. The classification of HSFs in wheat was based on the topology of the phylogenetic tree and the classification of HSFs in the other two plants.

Analysis of gene duplication and synteny relationships of TaHsfs

Segmental duplications and tandem duplications were determined for the TaHsfs using McscanX software [62]. Sequences on the same chromosome with a shared similarity greater than $75 \%$ and fragment lengths in the alignment that exceeded $75 \%$ of the length of the longer sequence were confirmed as tandem duplications. The KA/KS values were calculated for the tandem duplications using KAKS_caculator 2.0 [63]. A segmental duplication map of the genome was prepared using Circos software [64]. In addition to rice and Arabidopsis thaliana, the genome-wide CDS sequences and genome annotation information for Chinese cabbage

(http://brassicadb.org/brad/datasets/pub/Genomes/Brassica_rapa/V1.0/Scaffold1.0/), sorghum (http://www.plantgdb.org/BdGDB/), maize (http://interface.maizegdb.org/ gbrowse/maize_v4), and Brachypodium (https://phytozome.jgi.doe.gov/pz/portal. html\#!info?alias=Org_BdistachyonBd21_3_er) were obtained, and the synteny relationships between the HSF family in wheat and these species were determined using Mcscanx.

Structural characterization, conserved motifs, cis-elements, and construction of the interaction network for TaHsfs

ClustalW was used to align the conserved DBD and HR-A/B domains in the TaHsfs. The gene structures of the TaHsfs were visualized using the online drawing tool Gene Structure Display Server 2.0 (GSDS, http://gsds.cbi.pku.edu.cn/) [65]. The MEME software installed in the Linux system obtained 15 conserved motifs in the TaHsfs with lengths of 6-50 aa. The gene structure and motifs in the TaHsfs were determined using TB tools [66]. Sequences located 1500 bp upstream of the TaHsfs were obtained and the cis-acting elements were predicted with PLACE (plant cis-acting regulatory element, https://sogo.dna.affrc.go.jp/cgi-bin/sogo.cgi?sid=\&lang=en\&pj= 640\&action= page\&page=newplace) [67], and the results were displayed using GSDS. STRING (https://version-10-5.string-db.org/cgi/input.pl? sessionld= bHwqHDUnAsoF\&input_page_show_search=on) and PlantRegMap (http://plantregmap.cbi.pku.edu.cn/network.Php) were employed to obtain the regulation networks between TaHsfs and their interacting genes, which were visualized with Cytoscape (https://cytoscape.org/) [68-70].

RNA-seq data analysis

Part of the wheat transcriptome data was obtained from the Wheat Expression Browser powered by expVIP (http://www.wheat-expression.com/) to study the expression patterns of the TaHsfs. The RNA-seq data were derived from nine different growth periods and tissues in Chinese Spring under normal 
conditions (grain in reproductive stage, leaves and shoots in seedling stage, roots in seedling stage, leaves and shoots in vegetative stage, spike in reproductive stage, spike in vegetative stage, leaves and shoots in reproductive stage, roots in vegetative stage, and roots in reproductive stage) [71], as well as under six types of stress and a normal control in the heat and drought tolerant wheat variety TAM107 (combined drought and heat stress for $6 \mathrm{~h}$, combined drought and heat stress for $1 \mathrm{~h}$, heat stress for $6 \mathrm{~h}$, heat stress for $1 \mathrm{~h}$, drought stress for $6 \mathrm{~h}$, drought stress for $1 \mathrm{~h}$, and no stress control) [72]. In addition, in order to study the mechanism responsible for regulating TaHsfs in thermosensitive male sterile wheat, transcriptome data were analyzed from a type of temperature-sensitive male sterile wheat KTM3315A which was selected by our research group in 2001. KTM3315A exhibits male sterility under low temperature conditions $(<18 \mathrm{C})$ and fertility conversion under high temperature conditions $(>20 \mathrm{C})$ [49]. Ten pots of KTM3315A were placed in two artificial climate incubators before the fertility conversion period (binucleate stage) and they were treated at two different temperatures until the flowering stage. RNA was extracted from the anthers of sterile and fertile plants during the uninucleate, binucleate, and trinucleate stages (AS1, AS2, AS3, AF1, AF2, and AF3), and six samples were used for RNA-seq [49]. The heat maps were drawn using the heatmap application in omicshare (http://www.omicshare.com/tools/index.php/Home/help/index/ html/combinemore).

\section{Results}

Identification and basic characteristics of TaHsf genes

The newly constructed wheat-specific hidden Markov model (HMM) file was used to search the whole wheat protein sequences and 94 candidate Hsf genes were obtained, which were consistent with the wheat candidate Hsf genes obtained by alignments with Arabidopsis and rice Hsf genes. After identifying the DBD domain and coiled-coil structure, 61 non-redundant genes were finally identified in the wheat HSF family (Additional file 1: Table S1, Additional file 2: Table S2). These 61 TaHsfs were shown to be unequally distributed on 21 chromosomes (Additional file 3: Figure S1). The 5A chromosome contained the most TaHsf genes (6) and one to five TaHsfs were unevenly distributed on other chromosomes. We renamed these genes as TaHsf-01 to -61 according to their respective chromosome distribution. The gene name, transcript ID, gene position, exon number, aa length, molecular weight, and theoretical isoelectric point are listed for each of the TaHsfs in Table 1. The UniParct ID and old versions (IWGSC2.2) of the gene IDs are also included for clarity. The protein lengths of the TaHsfs ranged from 227 (TaHsf-22) to 569 (TaHsf-44), the molecular weights varied from 24.69 (TaHsf-22) to 59.76 (TaHsf-44), and the predicted isoelectric points ranged from 4.85 (TaHsf-12) to 9.52 (TaHsf-43).

Phylogenetic analysis of TaHsfs

The full-length amino acid sequences of $25 \mathrm{Hsf}$ genes in rice, 22 Hsf genes in Arabidopsis, and $61 \mathrm{Hsf}$ genes in wheat were obtained to co-construct a phylogenetic tree to classify the TaHsfs (Figure 1). Based on the clustering results, 61 TaHsfs were classified into three major categories: A, B, and C. The TaHsfs in class $A$ belonged to six subclasses of $A 1-A 6$, the class $B$ TaHsfs were divided into three subclasses of 
B1, B2, and B4; and the class C TaHsfs were divided into two subclasses of C1 and C2. Compared with rice and Arabidopsis Hsf members, the TaHsf family lacked members belonging to A7, A8, A9, and B3. In every subclass, at least one OsHsf was found to be highly homologous with the Hsf genes in wheat, e.g., OsHsf-13 (A1) was highly homologous with TaHsf-46, TaHsf-32, and TaHsf-51 in A1; OsHsf-07 (A3) shared high homology with TaHsf-10, TaHsf-14, and TaHsf-07 in A3; OsHsf-04 (A4b) in A4 was highly homologous with TaHsf-28, TaHsf-18, and TaHsf-13; and OsHsf-06 (A5) and TaHsf-52, TaHsf-53, TaHsf54 , in A5 were clustered together due to their high homology. OsHsf-17 (A6a) in A6, OsHsf-23 (B1) in B1, OsHsf-14 (B2a) and OsHsf-24 (B2C) in B2, OsHsf-10 (B4d) in B4, OsHsf-O2 (C1b) and OsHsf-03 (C1a) in $\mathrm{C} 1$, and OsHsf-05 (C2a) and OsHsf-16 (C2b) in C2 were also highly homologous with some Hsfs in wheat. However, only TaHsf-01 in A4 and OsHsf-15(A4d), and TaHsf-13 in B4 and OsHsf-19 (B4d) were orthologous genes. No orthologous genes were detected in the HSF family members in Arabidopsis and wheat, which indicates that the genetic relationship between wheat and rice is closer than that between wheat and Arabidopsis. Nineteen pairs of paralogous genes were found among the TaHsfs. A2 had the most paralogous genes with five pairs, as well as three pairs in $\mathrm{C} 1$ and $\mathrm{B} 2$, two pairs in $\mathrm{C} 2$, and one pair in each of the other subclasses. In addition to the initial the roles of heat stress response, those paralogous genes confered new functions for wheat HSF family, which is very important for the functional differentiation of this gene family.

Synteny analysis for TaHsfs

The synteny between species is strongly related to their evolution and kinship. In order to compare the kinship between wheat and other species based on HSFs, we performed synteny analysis between wheat with Arabidopsis thaliana, Chinese cabbage, rice, maize, sorghum, and Brachypodium. The results showed that TaHsfs were distantly related to the Hsfs in Arabidopsis thaliana and Brassica rapa, where only two and three genes were syntenic genes, respectively (Figure 2A). By contrast, the number of syntenic genes between wheat and rice (25), sorghum (39), corn (30), and Brachypodium distachyon (34), which all belong to the Gramineae, were higher (Figure 2B, Figure 2C). Arabidopsis and Brassica rapa are both cruciferous, so their $21 \mathrm{Hsf}$ genes had synteny relationships (Figure 2D). Thus, the Hsf genes in plants from the same family were more closely related, thereby suggesting that the evolution of Hsfs is consistent with the evolutionary direction of the whole genome. To further investigate the evolutionary relationships in the TaHsf family, we performed synteny analysis within the TaHsf genome. Only two pairs of tandem duplications comprising TaHsf-20/ TaHsf-19 and TaHsf-30/ TaHsf-29 occurred in the TaHsf family on the 3A and 3D chromosomes, respectively (Additional file 3: Figure S1), and their KA/KS values were 0.367731 and 0.388683 . Therefore, these two pairs of genes were subject to purifying selection. In addition, 52 TaHsfs participated in segmental duplication (Additional file 4: Table S3) and one to five TaHsfs were involved in each chromosome (Figure 3). The results showed that the probability of segmental duplication was greater for TaHsfs in the same chromosome than that between chromosomes. For instance, the segmental duplication on chromosome 2 only occurred between 2A, 2B, and 2D. Thus, the Hsf gene family in wheat mainly formed via segmental duplication.

Gene structure and motif composition in TaHsfs 
Multiple sequence alignments were performed to further analyze the degree of conservation in the DBD and OD domains in the TaHsfs. The results showed that the DBD amino acid lengths in the TaHsfs were 76-94 aa, and thus they were found to be highly conserved (Figure 4A). In particular, TaHsf-28 (76 aa) had a 17 aa deletion compared with most of the TaHsfs. The secondary structure of the DBD in the TaHsfs was predicted to comprise three alpha helices and four beta folds. According to the OD structure, the classification of the gene family was consistent with the results obtained by phylogenetic analysis, with 21 and seven aa insertions between the HR-A and HR-B in the class A and class C TaHsfs, respectively, and no insertion between the HR-A and HR-B in the class B TaHsfs (Figure 4B). The close phylogenetic relationships between the TaHsfs indicated the high evolutionary conservation of the ODs, and the sequences of the ODs in some pairs of paralogous genes were even identical. Figure 5 shows that the number of introns was very small in the TaHsf family members, ranging from 0 to 4 , and the lengths of the introns varied greatly. Motif prediction identified 15 conserved motifs with high confidence ranging between 11-50 aa in the TaHsf family, where motif 1 was the longest (50 aa) and motif 6 was the shortest (Additional file 5: Table S4). None of the TaHsf contained all 15 motifs. Three TaHsfs in subclass A1 contained the most motifs (10) and TaHsf-41 contained the least motifs (three). Motifs 1,2 , 5 , and 6 corresponded to the DBD domain, motif 3 and 4 to the OD domain, motif 7 to the NLS, and motif 10 to the AHA motif.

Predicted cis-elements in TaHsf promoters and interaction network analysis

In order to facilitate subsequent mining of the functions and mechanisms of TaHsfs, we predicted the ciselements in their upstream promoter regions (Figure 6). We screened six cis-elements comprising three related to stress responses (DRECRTCOREAT (dehydration-responsive element), CURECORECR (copperresponse element), and SORLIP1A (over-Represented in Light-Induced Promoters)) and the other three were CGCGBOXAT (Ca++/calmodulin binds), MYBPLANT (plant MYB binding site), and POLLEN1LELAT52 (one of two co-dependent regulatory elements responsible for pollen specific activation). The results showed that except TaHsf-14, there was at least one cis-element binding site in each TaHsf. The combination of TaHsf with DRECRTCOREAT, CURECORECR, and SORLIP1A also showed that TaHsfs function in the resistance to heat stress, but some TaHsfs also respond to drought, copper, and light stresses. The $\mathrm{Ca}++$ /calmodulin pathway is the primary signaling pathway and almost every TaHsf gene was shown to contain more than one binding site. In our previous study, we found that TaHsf interacts with MYB, and this result was confirmed by the MYBPLANT binding sites in TaHsf-15(B2), TaHsf-16 (C1), TaHsf-28 (A4), and TaHsf-35 (A2). Cis-element analysis allowed us to obtain the upstream factors that regulate their activity, but we determined their interactions using PlantReg Map (Figure 7A). The predicted results indicated interactions between $10 \mathrm{TaHsfs}$, including self-regulation between TaHsf02 and TaHsf-44, and both these and nine other genes had regulatory or regulated effects. In addition, we predicted their interacting genes using STRING and found that three main types of genes had interaction relationships with most of the TaHsfs, i.e., the target genes of TaHsfs comprised Hsp70s and Hsp90s, as well as the chaperone protein htpG (high-temperature protein G) (Figure 7B).

Expression profiles of TaHsfs 
In order to fully understand the expression patterns of the TaHsfs during each plant growth period and their organization, we analyzed RNA-seq data from the roots, stems, and leaves in the seedling, vegetative growth, and reproductive growth stages, as well spikes from the vegetative growth and reproductive growth stages, and the mature grain (Wheat Expression Browser, http://www.wheat-expression.com/, developmental time-course of Chinese Spring). The results showed that in the roots during the seedling stage, only TaHsfs in subclasses A2 (TaHsf-39 and TaHsf-49), C1 (TaHsf-16), and C2 (TaHsf-25, TaHsf36, and TaHsf-34) were upregulated. In the roots during the vegetative growth and reproductive growth stages, the TaHsfs with upregulated expression belonged to seven subclasses comprising A1(TaHsf-46, TaHsf-32, and TaHsf-51), A4 (TaHsf-23, TaHsf-18, TaHsf-28, and TaHsf-01), A5 (TaHsf-52, TaHsf-53, and TaHsf-54), B1 (TaHsf-37, TaHsf-43, and TaHsf-47), B2 (TaHsf-38 and TaHsf-44), C1 (TaHsf-16), and C2 (TaHsf-25, TaHsf-36, TaHsf-34, and TaHsf-42). In the stems and leaves during the seedling stage, the upregulated genes only belonged to subclasses A2 (TaHsf-05) and A3 (TaHsf-56). In the stems and leaves during the vegetative growth period, the upregulated genes belonged to subclasses A2 ( $T a H s f-03$ and TaHsf-04) and C1 (TaHsf-20, TaHsf-24, and TaHsf-29). In the stems and leaves during the reproductive growth period, the upregulated genes belonged to subclasses A2 (TaHsf-40, TaHsf-31, TaHsf-33, TaHsf-35, TaHsf-02, TaHsf-03, TaHsf-04, TaHsf-39, and TaHsf-49) and B2 (TaHsf-55, TaHsf-57, TaHsf-59, TaHsf-15, TaHsf-08, TaHsf-11, TaHsf-38, TaHsf-44, and TaHsf-48). In the spikes during the vegetative growth phase, the upregulated genes mainly belonged to subclasses B4 (TaHsf-06, TaHsf-13), A2 (TaHsf-45), A6 (TaHsf-07, TaHsf-10, TaHsf-14), and A5 (TaHsf-54). In the spikes during the reproductive growth phase, the upregulated TaHsfs mainly belonged to C1 (TaHsf-17, TaHsf-19, TaHsf-22, TaHsf-27, TaHsf-29, and TaHsf-21) and A2 (TaHsf-50, TaHsf-45, TaHsf-40, TaHsf-05, TaHsf-09, and TaHsf-12). Among the mature grains, the upregulated TaHsfs mainly belonged to subclasses A2 (TaHsf05, TaHsf-09, TaHsf-12, TaHsf-40, TaHsf-50), A3 (TaHsf-56, TaHsf-58), C2 (TaHsf-61, TaHsf-55, TaHsf-25, TaHsf-60), and A6 (TaHsf-14, TaHsf-07, TaHsf-10) (Figure 8A, Additional file 6: Table S5).

The analysis of cis-elements demonstrated that TaHsfs also regulate drought stress, so we investigated the expression patterns of TaHsfs under heat stress and drought stress (Wheat Expression Browser, http://www.wheat-expression.com/, drought and heat stress time-course in seedlings). The RNA-seq data showed that (Figure 8B, Additional file 7: Table S6) compared with the no stress control, the TaHsfs did not exhibit differences in their expression levels in response to drought stress for $1 \mathrm{~h}$, but the TaHsfs in $\mathrm{C} 1$ (TaHsf-16, TaHsf-21, TaHsf-17, TaHsf-22, TaHsf-26, and TaHsf-27) and C2 (TaHsf-60, TaHsf-36, TaHsf-61, TaHsf-25, and TaHsf-42) were significantly upregulated under drought stress for $6 \mathrm{~h}$. Under heat stress for $1 \mathrm{~h}$ and the combination of heat stress and drought stress for $1 \mathrm{~h}$, the expression levels of the TaHsfs were similar, where the main upregulated TaHsfs belonged to A2 (TaHsf-02, TaHsf-03, TaHsf-04, TaHsf31, TaHsf-39, TaHsf-49, TaHsf-33, TaHsf-35, TaHsf-50, TaHsf-45, and TaHsf-40), A3 (TaHsf-56 and TaHsf58), A4 (TaHsf-23, TaHsf-18, and TaHsf-28), A5 (TaHsf-52), B2 (TaHsf-08, TaHsf-15, TaHsf-11, TaHsf-57, TaHsf-55, and TaHsf-59), and B1 (TaHsf-37, TaHsf-43, and TaHsf-47). TaHsfs from A6 (TaHsf-50, TaHsf45, and TaHsf-40) and A4 (TaHsf-23, TaHsf-28, TaHsf-18) were upregulated under heat stress for $6 \mathrm{~h}$ and the combination of heat stress and drought stress for $6 \mathrm{~h}$, but TaHsfs from B2 (TaHsf-48, TaHsf-38, and 
TaHsf-44) were upregulated in the former conditions whereas TaHsfs from A6 (TaHsf-10, TaHsf-07, and TaHsf-14) were upregulated under the latter conditions.

In addition, in order to study the responses of TaHsfs in thermo-sensitive male sterile wheat to different fertility conditions, we analyzed the RNA-seq data for $40 \mathrm{TaHsfs}$ under two fertility conditions in three different periods. The results showed that compared with the sterile conditions, TaHsfs from A1 (TaHsf51, TaHsf-32, and TaHsf-46), A2 (TaHsf-50, TaHsf-02, TaHsf-04, TaHsf-05, TaHsf-09, TaHsf-35, TaHsf-12, and TaHsf-33), B1 (TaHsf-43 and TaHsf-47), and B2 (TaHsf-11 and TaHsf-55) were upregulated in the uninucleate anthers under fertile conditions. TaHsfs from A2 (TaHsf-12, TaHsf-33, TaHsf-45, and TaHsf50), A4 (TaHsf-18 and TaHsf-28), A5 (TaHsf-54), A6 (TaHsf-10), B1 (TaHsf-43), and B4 (TaHsf-06) were significantly upregulated in binucleate anthers, and TaHsfs from A2 (TaHsf-35, TaHsf-12, and TaHsf-33), A4 (TaHsf-07), A5 (TaHsf-53), and B2 (TaHsf-55) in the trinucleate anthers under fertile conditions. However, no TaHsfs in subclass $\mathrm{C}$ were upregulated under fertile conditions during these three periods (Figure 8C, Additional file 8: Table S7).

The results indicated that the responses of TaHsfs from different subclasses varied in different developmental stages, tissues, stresses, and ecological conditions, thereby demonstrating that the different subclasses of TaHsf have diverse functions and they may participate in different regulatory links and pathways.

\section{Discussion}

Wheat is an important food crop throughout the world and it is of great economic importance to increase its stress resistance, yield, and quality. The release of the wheat genome sequence had provided important information for identifying excellent agronomic traits, stress resistance genes, and heat resistance genes in wheat at the genome level, thereby facilitating the cultivation of high quality and stress-resistant (heat) wheat varieties. In plants, HSFs are involved in various molecular processes and they have essential roles in the responses to abiotic stresses. In this study, we identified the members of the Hsf gene family in wheat as well as investigating their evolutionary relationships, gene structure, expression levels under different conditions, and their expression in thermo-sensitive male sterile wheat under different fertility conditions. Our findings facilitate the subsequent study of the stress resistance mechanism in wheat and they provide a reference for understanding the molecular temperature sensing and regulation mechanisms in plants with temperature-sensitive biological characteristics.

Previous studies identified 21, 25, 25, 25, 25, and 22 Hsf genes in Arabidopsis, rice, maize, sorghum, tomato, and chickpea, respectively [12-15,34]. These findings suggest that the number of the plant HSF family members is about 25 and that the number of genes in this family is highly conserved. However, in the present study, we searched for wheat Hsfs using various methods and finally identified 61 wheat HSFs with DBD structures. The number of HSF family members appears to be unrelated to the genome size, e.g., 35 Hsfs were found in Chinese cabbage (485 Mb) and 137 Hsfs in pear (527 Mb) [20, 35]. In the present study, in addition to employing two approaches for identifying TaHsfs, we also used the wheat 
HSF sequence in the plantTFDB database and NCBI as the query sequence for aligning all of the wheat protein sequences. Verification using Pfam, SMART, Conserved Domain Database (CDD), MARCOIL, and other databases showed that the final results were exactly the same as those obtained using the two approaches employed in the present study. Thus, the method employed for constructing wheat-specific HSF HMM files with HSF HMM files (PF00447) in the present study was highly effective and reasonable, and the combination of multiple approaches avoided the failure to identify TaHsfs. However, after checking the wheat HSFs provided in plantTFDB, we found that several transcripts designated as HSFs by PlantTFDB had a DBD but they lacked the necessary coiled-coil structure for a HSF. These discrepancies may be explained as follows. First, the tools used for identification may have been different or the same tool could have been used with different parameter settings, e.g., changing the E-value threshold will affect the HSF screening results. Second, a different version of the genome could have been employed with different genome annotations, and thus the screening results obtained may have been different.

A gene family is a group of genes derived from the same ancestor and produced by a gene or by two or more copies of the gene. Orthologs are proteins that have evolved from a vertical lineage (species formation) of different species and they usually retain the same function as the original protein. A paralog is a protein derived from gene duplication in a certain species and it may develop new related functions [36]. In our study, the phylogenetic tree was constructed using the maximum likelihood method for similar regions in the wheat, rice, and Arabidopsis thaliana genomes. We detected two pairs of orthologous genes between TaHsfs and OsHsfs but 19 paralogous pairs, which indicate that the Hsfs gene in wheat was constructed before the differentiation of wheat and rice, and it expanded during the evolution of the respective species. The new functions of the wheat HSF family members mainly developed after differentiation. This phenomenon has also been verified widely in studies of other gene families in plants [37-39]. In contrast to the HSF clusters in rice and Arabidopsis, the wheat HSF family lacks the $A 7, A 8, A 9$, and $B 3$ subclasses, thereby indicating that gene deletion occurred in the wheat HSF family during its evolution. Two important events may occur during the evolution of genes: gene duplication and gene synteny. Synteny refers to the fact that two or more markers on a chromosome or chromosome segment from a species are located on homologous chromosome fragments in another species, but the relative order of these markers may vary, even within the same species. In this study, we analyzed the synteny relationships between wheat and Arabidopsis thaliana, Chinese cabbage, maize, rice, sorghum, and Brachypodium. The results showed that the syntenic relationships of Hsfs were stronger in species when the relationship with wheat was closer. Segmental duplication and tandem duplication are the two main causes of the expansion of gene families in plants [39]. After the occurrence of gene duplication, one of the genes exerts its original function and the other copy can accumulate mutations without a loss of function, thereby allowing the generation of new genes and regulatory networks, which can evolve new functions and network regulation mechanisms to allow plants to adapt better to the environment [40]. We found two pairs of tandem duplications and 59 pairs of segmental duplications in the HSF family in wheat, and thus segmental duplication has played a major role in the expansion of the HSF family in wheat. Similar to gramineous plants, the genes in Arabidopsis and maize 
are more prone to genome-wide replication and segmental duplication, and these are the main modes of gene expansion in wheat.

The evolution of gene families is often accompanied by an increase or loss of exons. The structural differences between introns and exons are often important, and they have played key roles in the evolution of gene families [41]. We analyzed the number and distribution of exons and introns in each member of the TaHsfs family. We found that the 61 TaHsfs contained 1-3 exons and 0-4 introns. The lengths and positions of the TaHsf gene introns in the same subclass were relatively well conserved, but the introns varied greatly among the different TaHsf subclasses. The presence of introns allows a variety of splicing modes and the same DNA sequence can produce different protein products via alternative splicing of the gene after transcription [42]. This is one of the explanations for the functional diversity of the wheat HSF family during evolution and it is also a cause of the different temperature responses of TaHsfs [43]. The sizes of HSF sequences have differed during the evolution of eukaryotes but their basic structure is highly conserved. In our study, motifs $1,2,5$, and 6 corresponded to the DBD domain located at the N-terminus of each TaHsf and their structures were found to be highly conserved, thereby making them highly compatible with HSEs to ensure the accuracy localization of the HSEs and the precise regulation of downstream gene expression $[7,44]$. Motifs 3 and 4 corresponded to the OD domain and plant HSFs can be divided into three categories based on the unique features of the OD: class A, class B, and class $C[5,45]$. This is one of the reasons why we excluded candidate TaHsfs without a coiled-coil structure but a DBD structure.

The heat shock response is an extremely complex regulatory process that involves multiple signals and genes. Plants have been shown to respond to high temperature stress mainly via the HSF-HSP pathway, calcium-calmodulin (Ca++-CaM) pathway, reactive oxygen species, and hormonal pathways [46]. The plant plasma membrane receives the heat stress signal and the fluidity of the cell phospholipid bilayer increases, thereby allowing a large amount of $\mathrm{Ca}++$ to flow into the cell. Next, the effect of the inflow of $\mathrm{Ca}++$ is amplified by a signaling cascade to transmit the heat stress signal to the nucleus, which leads to the expression and accumulation of HSPs, osmoregulatory proteins, and antioxidant proteins, thereby alleviating the damage caused by high temperature and making the plant heat resistant [47, 48]. MYB is one of the largest families of plant transcription factors involved in the regulation of cell differentiation during the cell cycle as well as responding to hormones and the environment. In our previous studies, we showed that MYBs were significantly upregulated under high temperature conditions and they interacted with TaHsfs [49]. Therefore, the cis-elements of the TaHsfs promoter region were predicted. Except for TaHsf-12, TaHsf-14, and TaHsf-24, we predicted at least one CGCGbox as a Ca-binding site for each gene. Moreover, each class of TaHsfs was shown to have binding sites for MYB.

In the present study, we analyzed the expression patterns of TaHsf genes in nine different developmental stages and various tissues in order to classify their tissue-specific expression. The results showed that the TaHsf expression patterns were similar in the roots where they were mostly upregulated during the vegetative growth and reproductive growth stages. TaHsf subclasses A1, A4, A5, B1, B2, C1, and C2 all exhibited upregulated expression in the roots during these stages, but the expression levels of the 
subclass A2 genes were not significantly upregulated, whereas these genes were upregulated in various tissues during other periods. In rice, the expression levels of A class Hsfs also vary in different specific tissues under normal conditions $[23,50]$. The functions of genes that participate in regulation can be predicted according to the preferential expression levels of genes under different environmental conditions in the same tissue. The overexpression of Hsfs can increase the tolerance of heat in plants by increasing the expression of Hsps and other related genes. Our analysis showed that class A and B TaHsfs were significantly upregulated under heat stress whereas class $\mathrm{C}$ TaHsfs were not, as also found in maize and pepper $[13,18]$. Under drought stress, the expression levels of class A and B TaHsfs were not significantly increased, but the $\mathrm{C}$ class TaHsfs were significantly upregulated, and thus it appears that the $\mathrm{A}$ and $\mathrm{B}$ class TaHsfs are involved in heat shock regulation whereas the $\mathrm{C}$ class TaHsfs are involved in drought regulation. Previous studies have shown that B1, B2, and B4 subclass TaHsfs are often induced by the heat shock reaction, and they may act as synergistic factors that allow A class TaHsfs to resist heat more effectively [29, 51, 52]. However, B3 subclass TaHsfs are downregulated as suppressors under heat shock conditions [18]. No A3 subclass TaHsfs were identified in wheat, so we could only check the coordinated expression of B1, B2, and B4 subclass TaHsfs and class A TaHsfs in response to heat. In our previous studies, we suggested that some Hsfs may be involved in pollen development in plants, which is related to fertility. In the present study, we showed that TaHsfs were specifically expressed under different fertility conditions, and that class A and B TaHsfs were upregulated under fertile conditions, and they were the same as the genes involved in the heat stress response. We originally considered that heat would induce the expression of these TaHsf genes and that their upregulated expression would lead to the upregulation of other fertility-related genes, thereby ensuring fertility. However, we found that some genes had pollen-specific expression-related binding sites based on our predictions of the cis-elements, and previous studies have shown that the AT2G26150 and AT4G13980 genes are critical during pollen reproduction $[53,54]$. Our analysis of the TaHsfs related to wheat fertility provides a basis for future investigations of the important roles of TaHsfs in the fertility regulation mechanism.

\section{Conclusions}

In this study, we comprehensively analyzed the HSF family in wheat based on the latest release of the complete wheat reference genome. We identified 61 TaHsfs and they were unevenly distributed on 21 chromosomes. According to phylogenetic comparisons with Arabidopsis and rice, and structural analysis of the gene products, the wheat HSFs were divided into three classes (A, B, and $C$ ) and 11 subclasses (A1-A6, B1, B2, B4, C1, and C2). The synteny relationships showed that the HSF family in wheat is closely related to that in Brachypodium, and the expansion of the wheat HSF family mainly occurred by segmental duplication. The 61 wheat Hsfs contain 0-4 introns and 1-3 exons, and the gene structures are highly conserved in the same subclass. Cis-element prediction and RNA-seq analysis showed that the different members of the TaHsf family have diverse roles. In addition to resisting heat stress, TaHsfs also participate in the regulation of other stresses such as drought. Analysis of the expression levels in temperature-sensitive male sterile wheat confirmed that some TaHsfs are associated with pollen development and fertility conversion in wheat. Thus, we identified new members of the wheat HSF family, 
thereby facilitating further research into the functions of HSFs and their roles in the temperature response mechanism in temperature-sensitive wheat.

\section{Abbreviations}

HSF: Heat shock transcription factor

Hsf: HSF gene

TaHsf: Wheat Hsf

HSE: Heat shock element

HSP: Heat shock protein

Hsp: HSP gene

DBD: DNA binding domain

OD: Oligomeric domain

NLS: Nuclear localization signal

NES: Nuclear export signal

CTAD: C-terminal activation domain

H2-T-H3: Helix-turn-helix

HR-A/B: Hydrophobic heptad repeat regions A and B

AHA: Acidic amino acid

HMM: Hidden Markov model

aa: Amino acid

DRECRTCOREAT: Dehydration-responsive element

CURECORECR: Copper-response element

SORLIP1A: Over-Represented in Light-Induced Promoters

CGCGBOXAT: $\mathrm{Ca}++/$ calmodulin binding region

MYBPLANT: Plant MYB binding site 
POLLEN1LELAT52: One of two co-dependent regulatory elements responsible for pollen specific activation

htpG: High-temperature protein G

CDD: Conserved domain database

HSF-HSP: Heat stress transcription factor-heat shock protein

Ca++-CaM: Calcium-calmodulin

\section{Declarations}

Competing interests

The authors declare that they have no competing financial interests.

Authors' contributions

Xiyue Song and Lingli Zhang designed the research. Jiali Ye and Xuetong Yang performed the experiments. Jiali Ye, Sha Li, Qi Liu, and Wei Li analyzed the data. Jiali Ye wrote the manuscript.

\section{Acknowledgemnts}

This study was sponsored by the National Natural Science Foundation of China (31771874) and the Program in Science and Technology of Yangling State Demonstration Zone of Agricultural High-tech Industries (2018NY-19).

\section{References}

1. Kumar SV, Wigge PA: H2A.Z-containing nucleosomes mediate the thermosensory response in Arabidopsis. Cell 2010, 140(1):136-147.

2. Sung D, Kaplan F, Lee K, Guy CL: Acquired tolerance to temperature extremes. Trends in Plant Science 2003, 8(4):179-187.

3. Parsell DA, Lindquist S: The function of heat-shock proteins in stress tolerance: degradation and reactivation of damaged proteins. Annual Review of Genetics 1993, 27(1):437-496.

4. Hartl FU, Hayerhartl M: Molecular chaperones in the cytosol: from nascent chain to folded protein. Science 2002, 295(5561):1852-1858.

5. Nover L, Bharti K, Doring P, Mishra SK, Ganguli A, Scharf K: Arabidopsis and the heat stress transcription factor world: how many heat stress transcription factors do we need? Cell Stress \& Chaperones 2001, 6(3):177-189. 
6. Damberger FF, Pelton JG, Harrison CJ, Nelson HCM, Wemmer DE: Solution structure of the DNA-binding domain of the heat shock transcription factor determined by multidimensional heteronuclear magnetic resonance spectroscopy. Protein Science 1994, 3(10):1806-1821.

7. Cicero MP, Hubl ST, Harrison CJ, Littlefield O, Hardy JA, Nelson HCM: The wing in yeast heat shock transcription factor (HSF) DNA-binding domain is required for full activity. Nucleic Acids Research 2001, 29(8):1715-1723.

8. Yura T, Nakahigashi K: Regulation of the heat-shock response. Current Opinion in Microbiology 1999, 2(2):153-158.

9. Scharf K, Berberich T, Ebersberger I, Nover L: The plant heat stress transcription factor (Hsf) family: Structure, function and evolution. Biochimica et Biophysica Acta 2012, 1819(2):104-119.

10. Heerklotz D, Doring P, Bonzelius F, Winkelhaus S, Nover L: The balance of nuclear import and export determines the intracellular distribution and function of tomato heat stress transcription factor HsfA2. Molecular and Cellular Biology 2001, 21(5): 1759-1768.

11. Mishra SK, Tripp J, Winkelhaus S, Tschiersch B, Theres K, Nover L, Scharf K: In the complex family of heat stress transcription factors, HsfA1 has a unique role as master regulator of thermotolerance in tomato. Genes \& Development 2002, 16(12):1555-1567.

12. Guo J, Wu J, Ji Q, Wang C, Luo L, Yuan Y, Wang Y, Wang J: Genome-wide analysis of heat shock transcription factor families in rice and Arabidopsis. Journal of Genetics and Genomics 2008, 35(2):105118.

13. Lin Y, Jiang H, Chu Z, Tang X, Zhu S, Cheng B: Genome-wide identification, classification and analysis of heat shock transcription factor family in maize. BMC Genomics 2011, 12(1):76-76.

14. Li P, Yu T, He G, Chen M, Zhou Y, Chai S, Xu Z, Ma Y: Genome-wide analysis of the Hsf family in soybean and functional identification of GmHsf-34 involvement in drought and heat stresses. $B M C$ Genomics 2014, 15(1):1009-1009.

15. Chidambaranathan P, Jagannadham PTK, Satheesh V, Kohli D, Basavarajappa SH, Chellapilla B, Kumar J, Jain PK, Srinivasan R: Genome-wide analysis identifies chickpea (Cicer arietinum) heat stress transcription factors (Hsfs) responsive to heat stress at the pod development stage. Journal of Plant Research 2018, 131(3):525-542.

16. Nagaraju M, Reddy PS, Kumar SA, Srivastava RK, Kishor PBK, Rao DM: Genome-wide scanning and characterization of Sorghum bicolor L. heat shock transcription factors. Current Genomics 2015, 16(4):279-291.

17. Ma J, Xu Z, Wang F, Tan G, Li M, Xiong A: Genome-wide analysis of HSF family transcription factors and their responses to abiotic stresses in two Chinese cabbage varieties. Acta Physiologiae Plantarum 
2014, 36(2):513-523.

18. Guo M, Lu J, Zhai Y, Chai W, Gong Z, Lu M: Genome-wide analysis, expression profile of heat shock factor gene family (CaHsfs) and characterisation of CaHsfA2 in pepper (Capsicum annuum L.). BMC Plant Biology 2015, 15(1):151-151.

19. Giorno F, Guerriero G, Baric S, Mariani C: Heat shock transcriptional factors in Malus domestica: identification, classification and expression analysis. BMC Genomics 2012, 13(1):639-639.

20. Qiao X, Li M, Li L, Yin H, Wu J, Zhang S: Genome-wide identification and comparative analysis of the heat shock transcription factor family in Chinese white pear (Pyrus bretschneideri) and five other Rosaceae species. BMC Plant Biology 2015, 15(1):12-12.

21. Lohmann C, Eggersschumacher G, Wunderlich M, Schoffl F: Two different heat shock transcription factors regulate immediate early expression of stress genes in Arabidopsis. Molecular Genetics and Genomics 2004, 271(1):11-21.

22. Busch W, Wunderlich M, Schoffl F: Identification of novel heat shock factor-dependent genes and biochemical pathways in Arabidopsis thaliana. Plant Journal 2004, 41(1):1-14.

23. Ogawa D, Yamaguchi K, Nishiuchi T: High-level overexpression of the Arabidopsis HsfA2 gene confers not only increased themotolerance but also salt/osmotic stress tolerance and enhanced callus growth. Journal of Experimental Botany 2007, 58(12):3373-3383.

24. Banti V, Mafessoni F, Loreti E, Alpi A, Perata P: The heat-inducible transcription factor HsfA2 enhances anoxia tolerance in Arabidopsis. Plant Physiology 2010, 152(3):1471-1483.

25. Zhang L, Li Y, Xing D, Gao C: Characterization of mitochondrial dynamics and subcellular localization of ROS reveal that HsfA2 alleviates oxidative damage caused by heat stress in Arabidopsis. Journal of Experimental Botany 2009, 60(7):2073-2091.

26. Giorno F, Woltersarts M, Grillo S, Scharf K, Vriezen WH, Mariani C: Developmental and heat stressregulated expression of HsfA2 and small heat shock proteins in tomato anthers. Journal of Experimental Botany 2010, 61(2):453-462.

27. Shim D, Hwang J, Lee J, Lee S, Choi Y, An G, Martinoia E, Lee Y: Orthologs of the class A4 heat shock transcription factor HsfA4a confer cadmium tolerance in wheat and rice. The Plant Cell 2009, 21(12):4031-4043.

28. Almoguera C, Prietodapena P, Diazmartin J, Espinosa JMR, Carranco R, Jordano J: The HaDREB2 transcription factor enhances basal thermotolerance and longevity of seeds through functional interaction with HaHSFA9. BMC Plant Biology 2009, 9(1):75-75. 
29. Bharti K, Von Koskulldoring P, Bharti S, Kumar P, Tintschlkorbitzer A, Treuter E, Nover L: Tomato heat stress transcription factor HsfB1 represents a novel type of general transcription coactivator with a histone-like motif interacting with the plant CREB binding protein ortholog HAC1. The Plant Cell 2004, 16(6):1521-1535.

30.Prasad PVV, Djanaguiraman M: Response of floret fertility and individual grain weight of wheat to high temperature stress: sensitive stages and thresholds for temperature and duration. Functional Plant Biology 2014, 41(12):1261-1269.

31.Xue G, Sadat S, Drenth J, McIntyre CL: The heat shock factor family from Triticum aestivum in response to heat and other major abiotic stresses and their role in regulation of heat shock protein genes. Journal of Experimental Botany 2014, 65(2):539-557.

32.Zhang S, Xu Z, Li P, Yang L, Wei Y, Chen M, Li L, Zhang G, Ma Y: Overexpression of TaHsf3 in transgenic Arabidopsis enhances tolerance to extreme temperatures. Plant Molecular Biology Reporter 2013, 31(3):688-697.

33. Kumar RR, Goswami S, Singh K, Dubey K, Rai GK, Singh B, Singh S, Grover M, Mishra D, Kumar S: Characterization of novel heat-responsive transcription factor (TaHsfA6e) gene involved in regulation of heat shock proteins (HSPs) -A key member of heat stress-tolerance network of wheat. Journal of Biotechnology 2018, 279:1-12.

34. Chen $\mathrm{Y}$, Wang $\mathrm{Y}$, Zhang $\mathrm{H}$ : Identification and expression analysis of heat shock factor (Hsf) gene family in tomato (Solanum lycopersicum). Journal of Agricultural Biotechnology 2015, 23(4):492-501.

35. Song X, Liu G, Duan W, Liu T, Huang Z, Ren J, Li Y, Hou X: Genome-wide identification, classification and expression analysis of the heat shock transcription factor family in Chinese cabbage. Molecular Genetics and Genomics 2014, 289(4):541-551.

36. Thornton JW, Desalle R: Gene family evolution and homology: genomics meets phylogenetics. Annual Review of Genomics and Human Genetics 2000, 1(1):41-73.

37. Bai J, Pennill LA, Ning J, Lee SW, Ramalingam J, Webb CA, Zhao B, Sun Q, Nelson JC, Leach JE: Diversity in nucleotide binding site-leucine-rich repeat genes in cereals. Genome Research 2002, 12(12):1871-1884.

38. Jain MK, Tyagi AK, Khurana JP: Genome-wide analysis, evolutionary expansion, and expression of early auxin-responsive SAUR gene family in rice (Oryza sativa). Genomics 2006, 88(3):360-371.

39. Cannon SB, Mitra A, Baumgarten A, Young ND, May G: The roles of segmental and tandem gene duplication in the evolution of large gene families in Arabidopsis thaliana. BMC Plant Biology 2004, 4(1):10-10. 
40. He X, Zhang J: Rapid subfunctionalization accompanied by prolonged and substantial neofunctionalization in duplicate gene evolution. Genetics 2005, 169(2):1157-1164.

41. Rose AB: Intron-mediated regulation of gene expression. Current Topics in Microbiology and Immunology 2008, 326:277-290.

42. Yuebing $\mathrm{W}$, Zhihong $\mathrm{L}$, Dafang $\mathrm{H}$ : Effects of pre-mRNA introns on regulation of eukaryotic gene expression. Biotechnology Bulletin 2008, 4.

43. Vu LD, Gevaert K, De Smet I: Feeling the heat: searching for plant thermosensors. Trends in Plant Science 2019, 24(3):210-219.

44. Peteranderl R, Rabenstein M, Shin Y, Liu CW, Wemmer DE, King DS, Nelson HCM: Biochemical and biophysical characterization of the trimerization domain from the heat shock transcription factor. Biochemistry 1999, 38(12):3559-3569.

45. Nover L, Scharf K, Gagliardi D, Vergne P, Czarneckaverner E, Gurley WB: The Hsf world: classification and properties of plant heat stress transcription factors. Cell Stress \& Chaperones 1996, 1(4):215-223.

46. Mittler R, Finka A, Goloubinoff P: How do plants feel the heat. Trends in Biochemical Sciences 2012, 37(3):118-125.

47. Reddy ASN, Ali GS, Celesnik H, Day IS: Coping with stresses: roles of calcium- and calcium/calmodulin-regulated gene expression. The Plant Cell 2011, 23(6):2010-2032.

48. Liu H, Liao H, Charng Y: The role of class A1 heat shock factors (HSFA1s) in response to heat and other stresses in Arabidopsis. Plant Cell and Environment 2011, 34(5):738-751.

49.Ye J, Duan Y, Hu G, Geng X, Zhang G, Yan P, Liu Z, Zhang L, Song X: Identification of candidate genes and biosynthesis pathways related to fertility conversion by wheat KTM3315A transcriptome profiling. Frontiers in Plant Science 2017, 8(449).

50. Liu A, Zou J, Zhang X, Zhou X, Wang W, Xiong X, Chen L, Chen X: Expression profiles of class A rice heat shock transcription factor genes under abiotic stresses. Journal of Plant Biology 2010, 53(2):142149 .

51. Czarneckaverner E, Yuan C, Scharf K, Englich G, Gurley WB: Plants contain a novel multi-member class of heat shock factors without transcriptional activator potential. Plant Molecular Biology 2000, 43(4):459-471.

52. Ikeda M, Mitsuda N, Ohmetakagi M: Arabidopsis HsfB1 and HsfB2b act as repressors of the expression of heat-inducible Hsfs but positively regulate the acquired thermotolerance. Plant Physiology 2011, 157(3):1243-1254. 
53. Reňák D, Gibalova A, Solcova K, Honys D: A new link between stress response and nucleolar function during pollen development in Arabidopsis mediated by AtREN1 protein. Plant Cell and Environment 2014, 37(3):670-683.

54. Tuncozdemir M, Tang C, Ishka MR, Brown E, Groves NR, Myers CT, Rato C, Poulsen LR, McDowell SC, Miller G: A cyclic nucleotidegated channel (CNGC16) in pollen is critical for stress tolerance in pollen reproductive development. Plant Physiology 2013, 161(2):1010-1020.

55. Investigators IRP, Appels R, Eversole K, Feuillet C, Keller B, Rogers J, Stein N, Investigators IWAP, Pozniak CJ, Choulet F: Shifting the limits in wheat research and breeding using a fully annotated reference genome. Science 2018, 361(6403).

56. Elgebali S, Mistry J, Bateman A, Eddy SR, Luciani A, Potter SC, Qureshi M, Richardson L, Salazar GA, Smart A: The Pfam protein families database in 2019. Nucleic Acids Research 2019, 47: D427-D432.

57. Marchlerbauer A, Bo Y, Han L, He J, Lanczycki CJ, Lu S, Chitsaz F, Derbyshire MK, Geer RC, Gonzales NR: CDD/SPARCLE: functional classification of proteins via subfamily domain architectures. Nucleic Acids Research 2017, 45.

58. Letunic I, Bork P: 20 years of the SMART protein domain annotation resource. Nucleic Acids Research 2018, 46: D493-D496.

59. Delorenzi M, Speed TP: An HMM model for coiled-coil domains and a comparison with PSSM-based predictions. Bioinformatics 2002, 18(4):617-625.

60. La Cour T, Kiemer L, Molgaard A, Gupta R, Skriver K, Brunak S: Analysis and prediction of leucine-rich nuclear export signals. Protein Engineering Design \& Selection 2004, 17(6):527-536.

61. Kosugi S, Hasebe M, Tomita M, Yanagawa H: Systematic identification of cell cycledependent yeast nucleocytoplasmic shuttling proteins by prediction of composite motifs. Proceedings of the National Academy of Sciences of the United States of America 2009, 106(25):10171-10176.

62. Wang Y, Tang H, Debarry JD, Tan X, Li J, Wang X, Lee T, Jin H, Marler BS, Guo H: MCScanX: a toolkit for detection and evolutionary analysis of gene synteny and collinearity. Nucleic Acids Research 2012, 40(7): e49.

63. Guo J, Jain R, Yang P, Fan R, Kwoh CK, Zheng J: Reliable and fast estimation of recombination rates by convergence diagnosis and parallel Markov Chain Monte Carlo. IEEE/ACM Transactions on Computational Biology and Bioinformatics 2014, 11(1):63-72.

64. Krzywinski M, Schein JE, Birol I, Connors JM, Gascoyne RD, Horsman D, Jones SJM, Marra MA: Circos: An information aesthetic for comparative genomics. Genome Research 2009, 19(9):1639-1645. 
65. Hu B, Jin J, Guo A, Zhang H, Luo J, Gao G: GSDS 2.0: an upgraded gene feature visualization server. Bioinformatics 2015, 31(8):1296-1297.

66. Chen $\mathrm{C}$, Xia R, Chen H, He Y: TBtools, a Toolkit for Biologists integrating various HTS-data handling tools with a user-friendly interface. bioRxiv 2018:289660.

67. Higo $\mathrm{K}$, Ugawa $\mathrm{Y}$, Iwamoto $\mathrm{M}$, Korenaga T: Plant cis-acting regulatory DNA elements (PLACE) database: 1999. Nucleic Acids Research 1999, 27(1):297-300.

68. Szklarczyk D, Morris JH, Cook H, Kuhn M, Wyder S, Simonovic M, Santos A, Doncheva NT, Roth A, Bork P: The STRING database in 2017: quality-controlled protein-protein association networks, made broadly accessible. Nucleic Acids Research 2017, 45:0.

69. Jin J, Tian F, Yang D, Meng Y, Kong L, Luo J, Gao G: PlantTFDB 4.0: toward a central hub for transcription factors and regulatory interactions in plants. Nucleic Acids Research 2017, 45.

70. Shannon P, Markiel A, Ozier O, Baliga NS, Wang JT, Ramage D, Amin N, Schwikowski B, Ideker T: Cytoscape: A software environment for integrated models of biomolecular interaction networks. Genome Research 2003, 13(11):2498-2504.

71. Clavijo BJ, Venturini L, Schudoma C, Accinelli GG, Kaithakottil G, Wright J, Borrill P, Kettleborough G, Heavens D, Chapman HD: An improved assembly and annotation of the allohexaploid wheat genome identifies complete families of agronomic genes and provides genomic evidence for chromosomal translocations. Genome Research 2017, 27(5):885-896.

72. Choulet F, Alberti A, Theil S, Glover N, Barbe V, Daron J, Pingault L, Sourdille P, Couloux A, Paux E et al: Structural and functional partitioning of bread wheat chromosome 3B. Science 2014, 345(6194):1249721.

\section{Tables}

Due to technical limitations, Table(s) 1 and 2 are only available as a download in the supplemental files section.

\section{Figures}




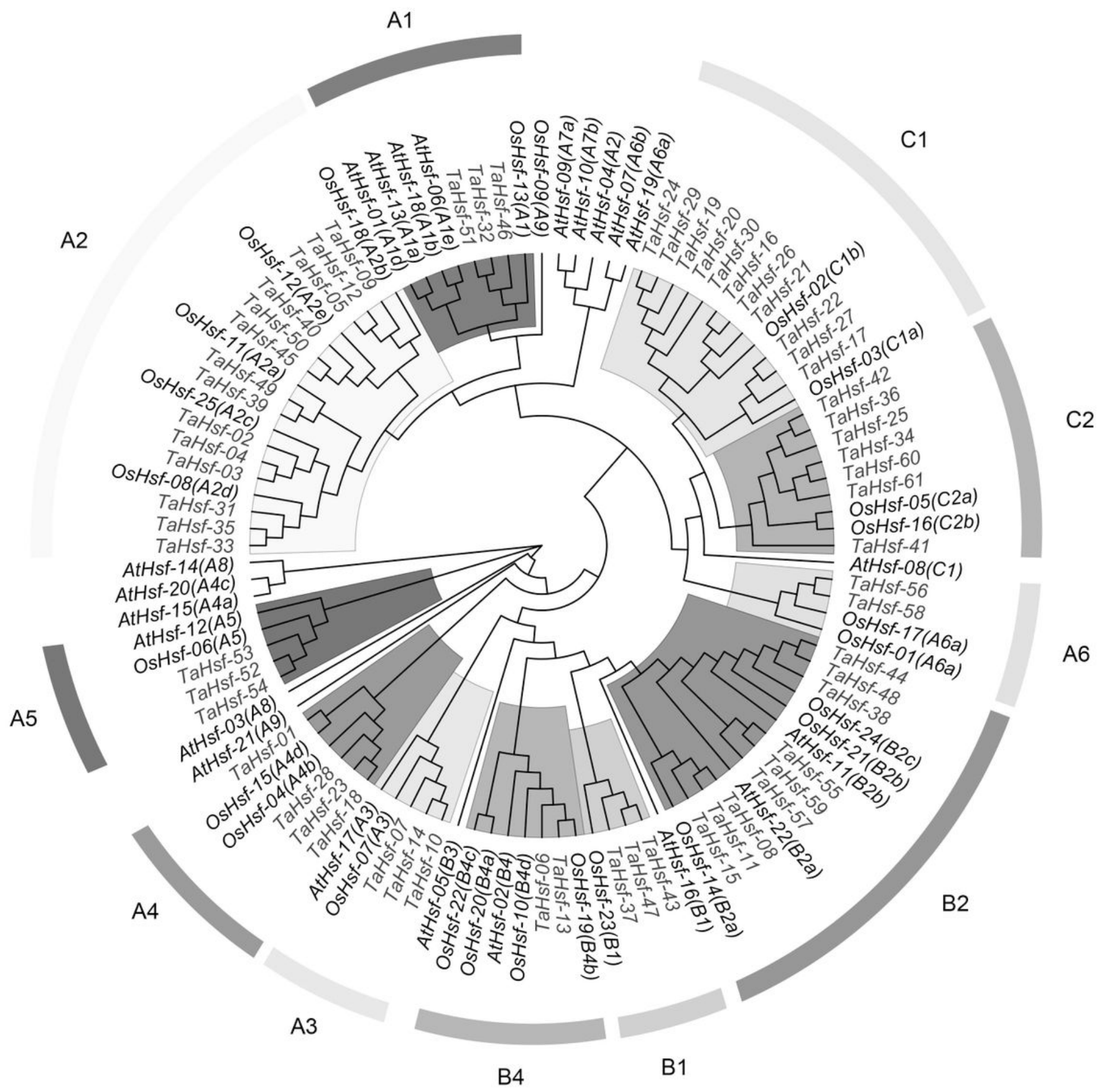

Figure 1

Phylogenetic tree obtained for the HSF family in wheat, rice, and Arabidopsis. 


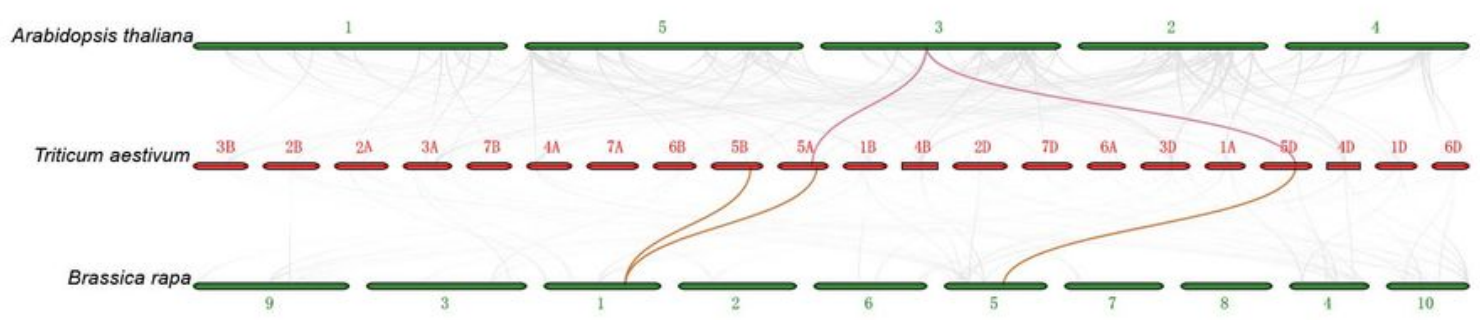

\section{B}

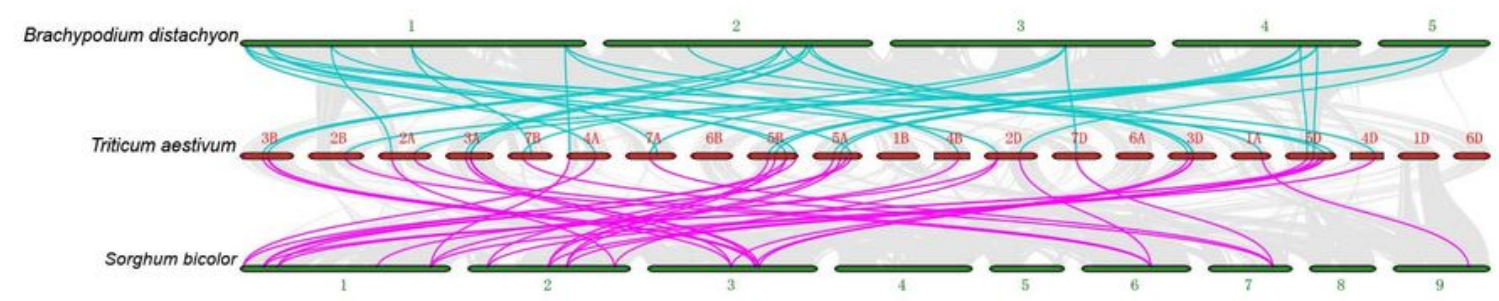

\section{$2 \mathrm{C}$}

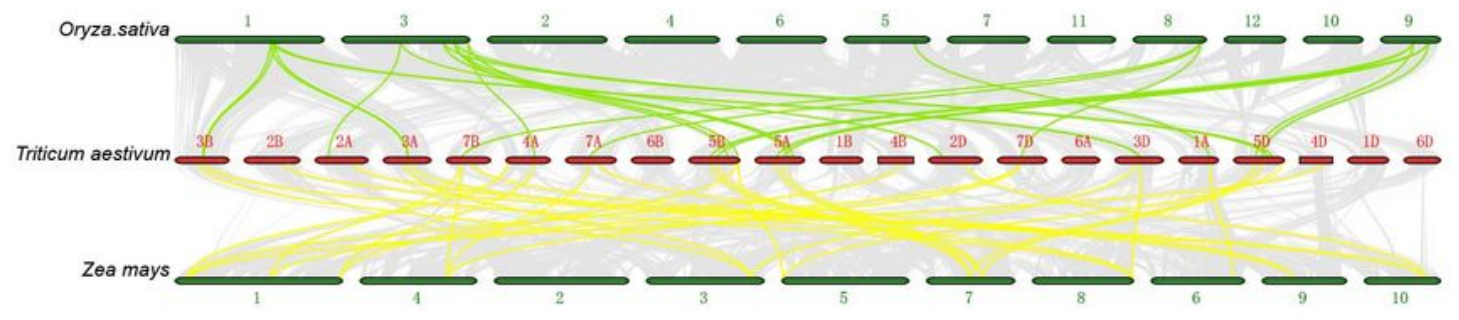

\section{D}

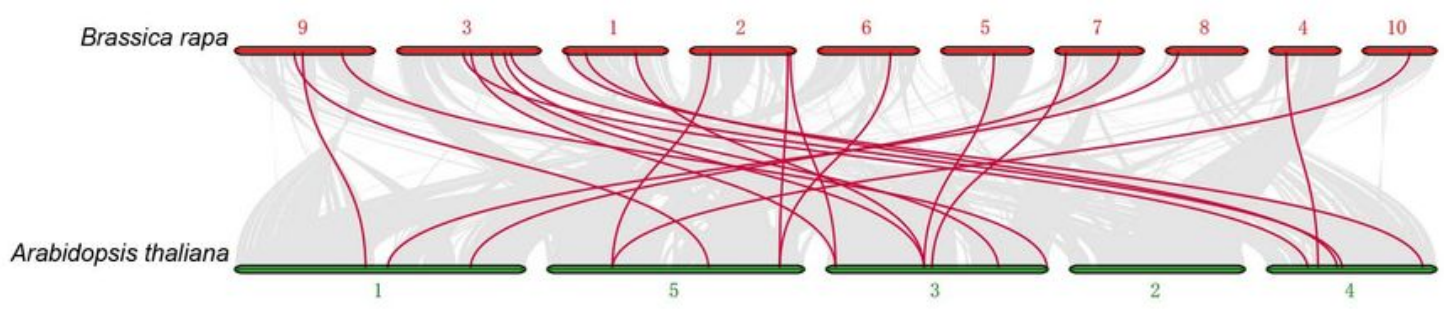

\section{Figure 2}

Synteny relationships of Hsfs in wheat and six representative species. Gray lines in the background indicate the synteny blocks for wheat and other plant genomes, and the lines in other colors highlight the synteny of Hsf gene pairs. 


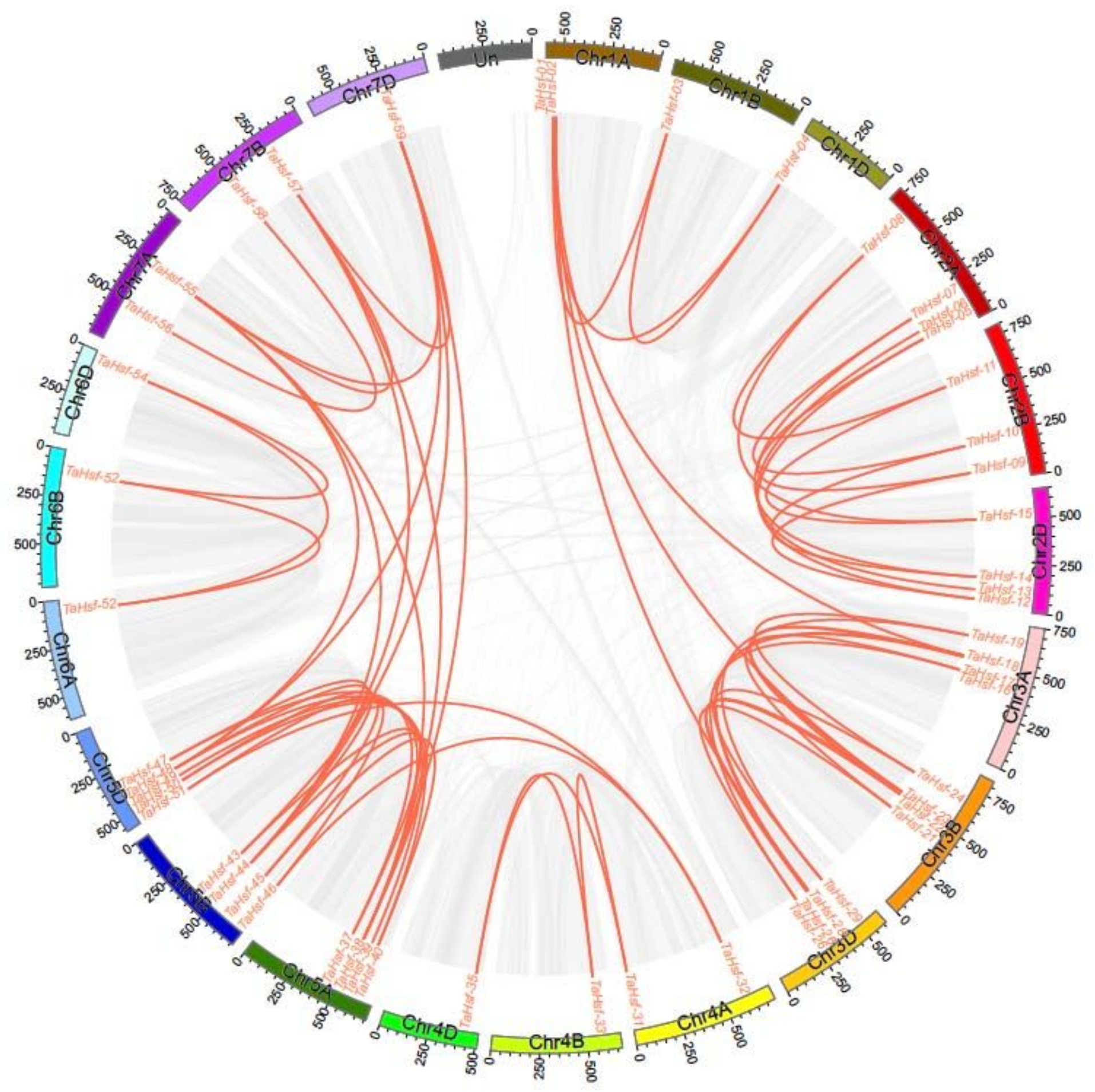

Figure 3

Genomic locations of Hsfs and duplicated gene pairs in the wheat genome. Gray lines in the background indicate the synteny blocks within the whole wheat genome, and red lines denote the segmental duplication Hsf gene pairs. 


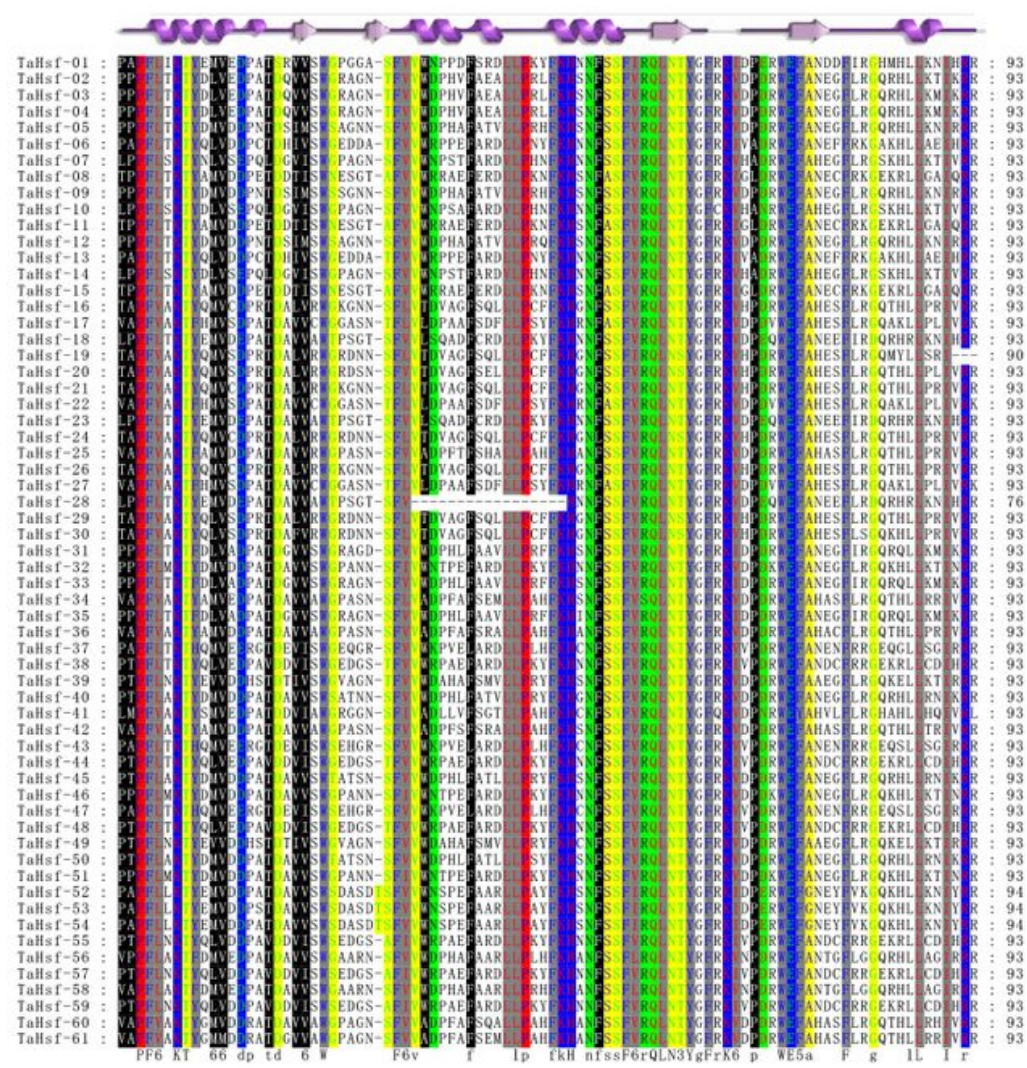

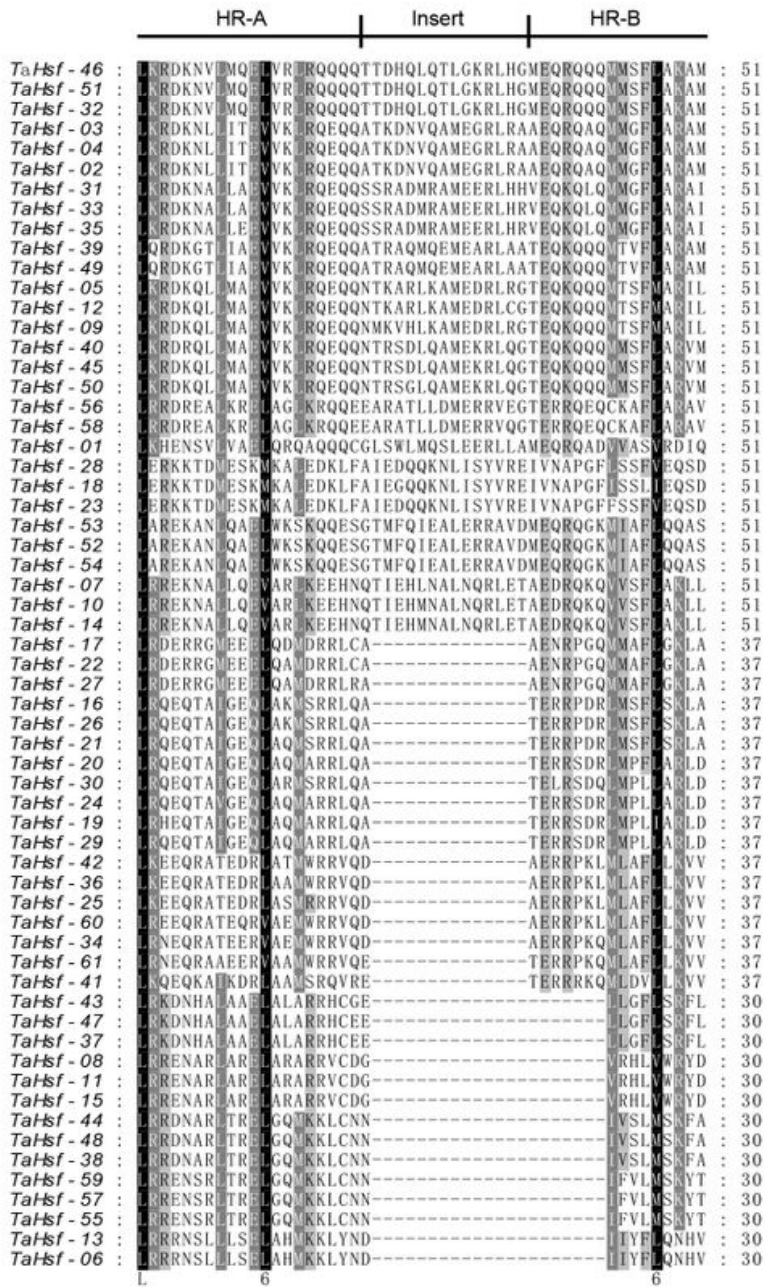

\section{Figure 4}

Analysis of the conservation of DBD domains and OD regions in the $61 \mathrm{Hsf}$ genes in wheat. a. Multiple sequence alignment of the DBD domains in 61 TaHsfs. The scheme at the top depicts the protein secondary structure predicted based on the conserved DBD domain. b. Multiple sequence alignment of the HR-A/B regions in 61 TaHsfs. The scheme at the top shows the locations and boundaries of the HR-A core, insert, and HR-B regions within the HR-A/B regions. 

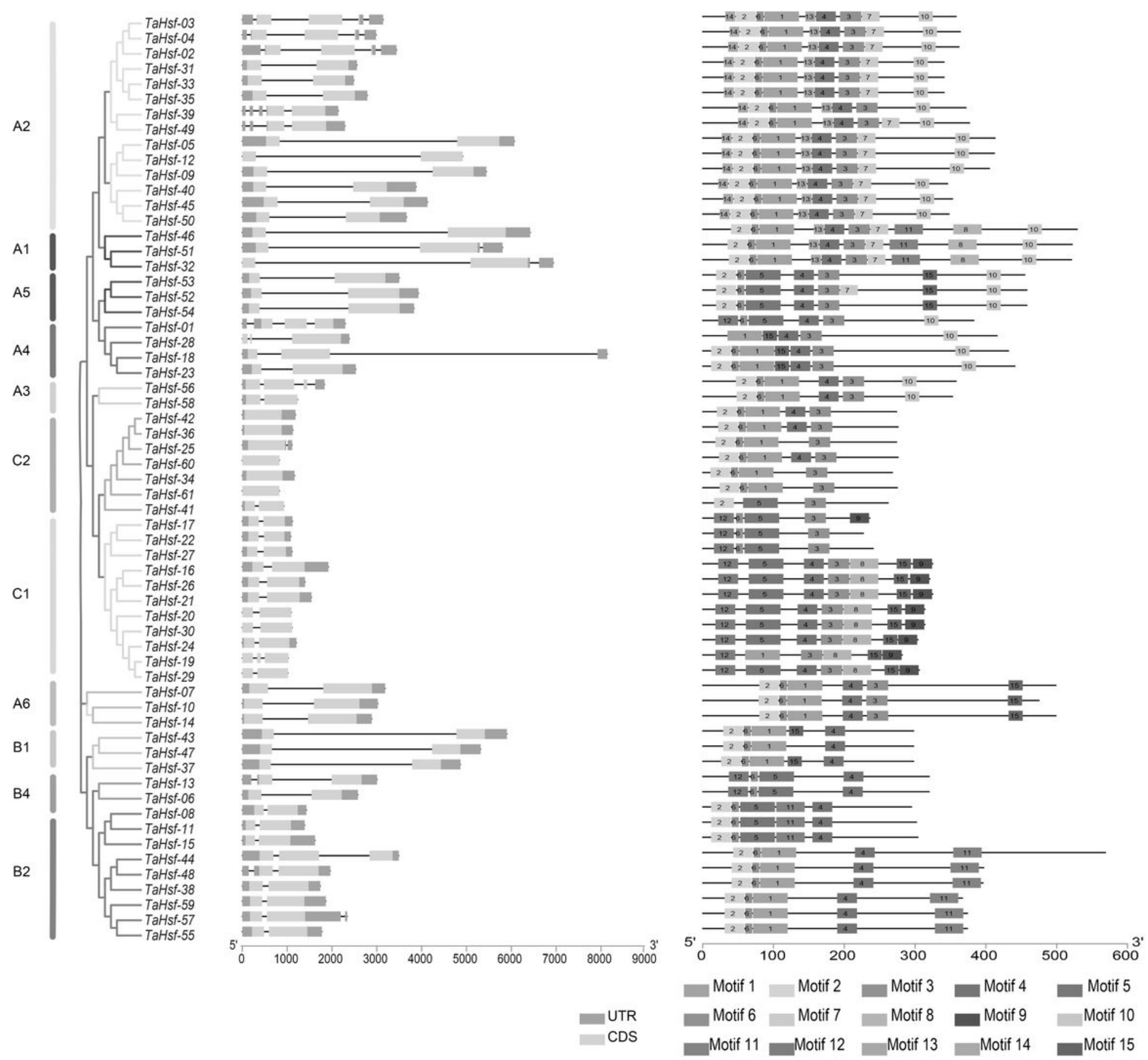

Figure 5

Phylogenetic tree, gene structure, and architecture of conserved protein motifs in TaHsfs. Details of the clusters in the phylogenetic tree are shown in different colors. The left panel represents the intron-exon structure of the TaHsfs, and the right panel shows the motif compositions of the TaHsfs. Different motifs are indicated by different colors and they are numbered from 1-15. 


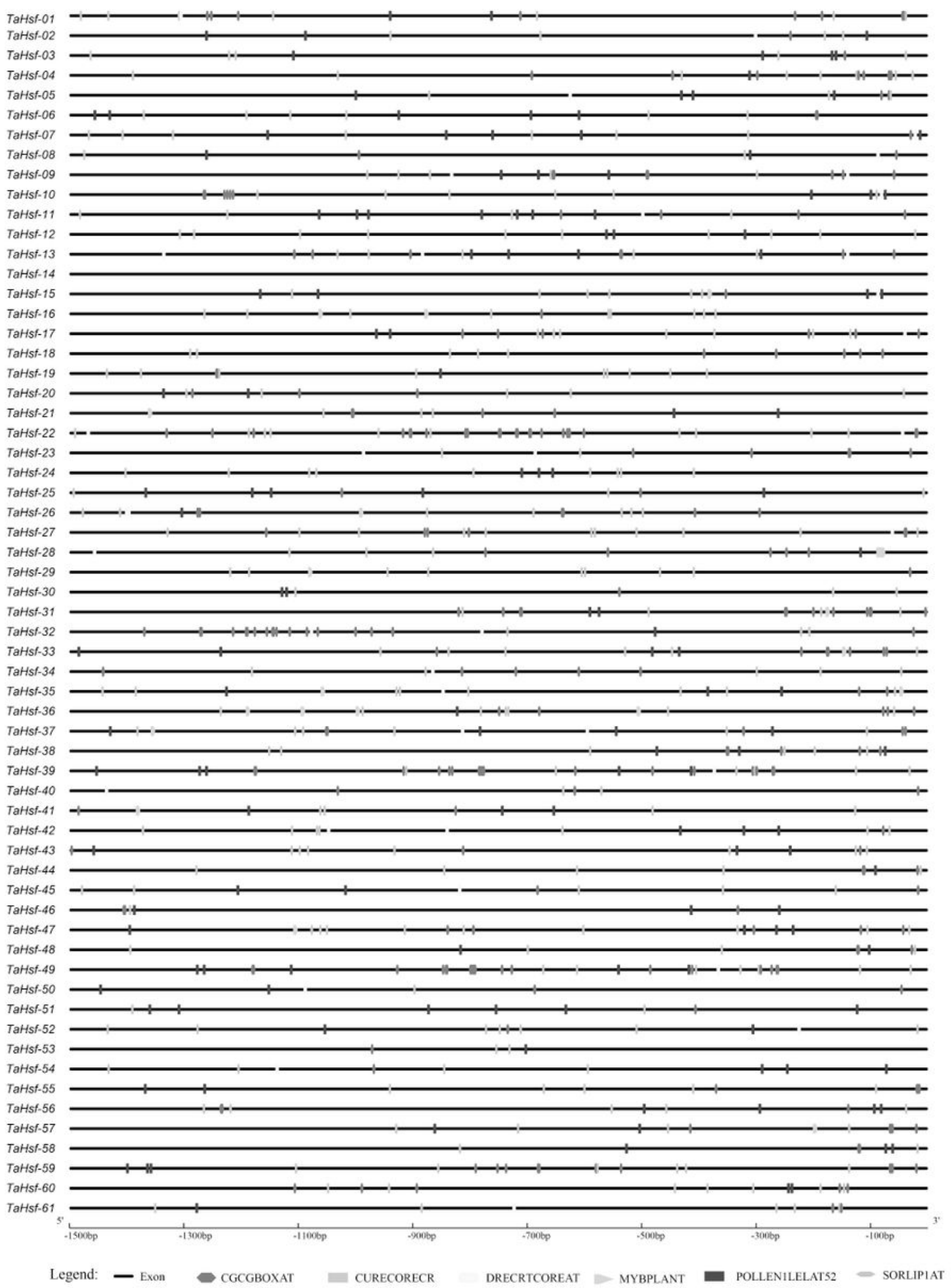

\section{Figure 6}

Distribution of cis-element binding sites in the promoter regions of Hsf family members in wheat. 


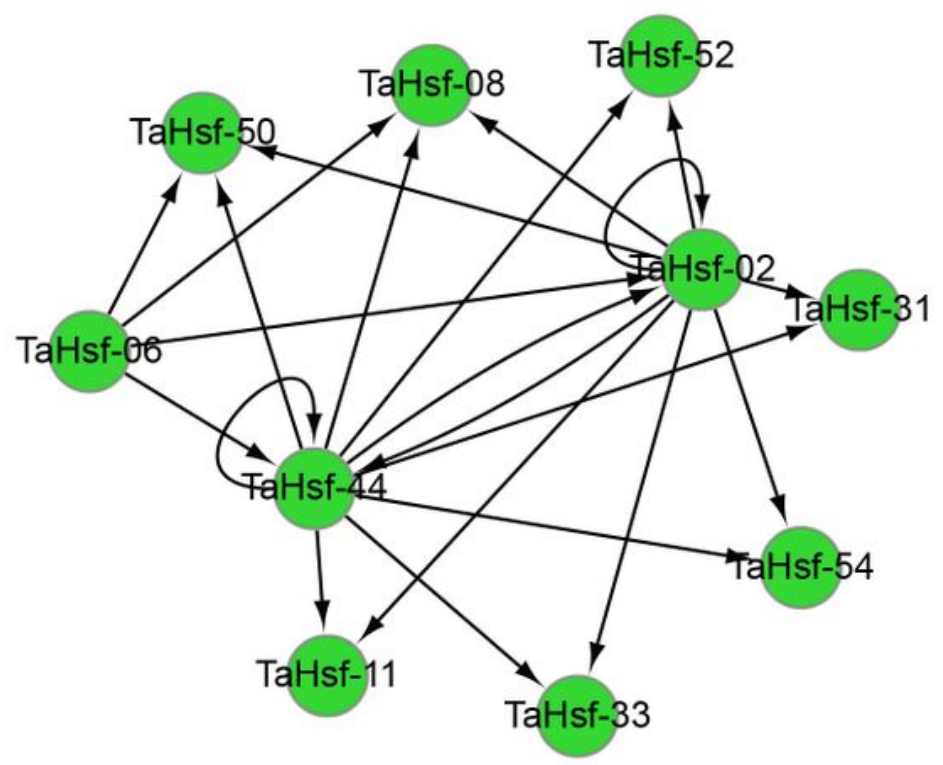

\section{B}

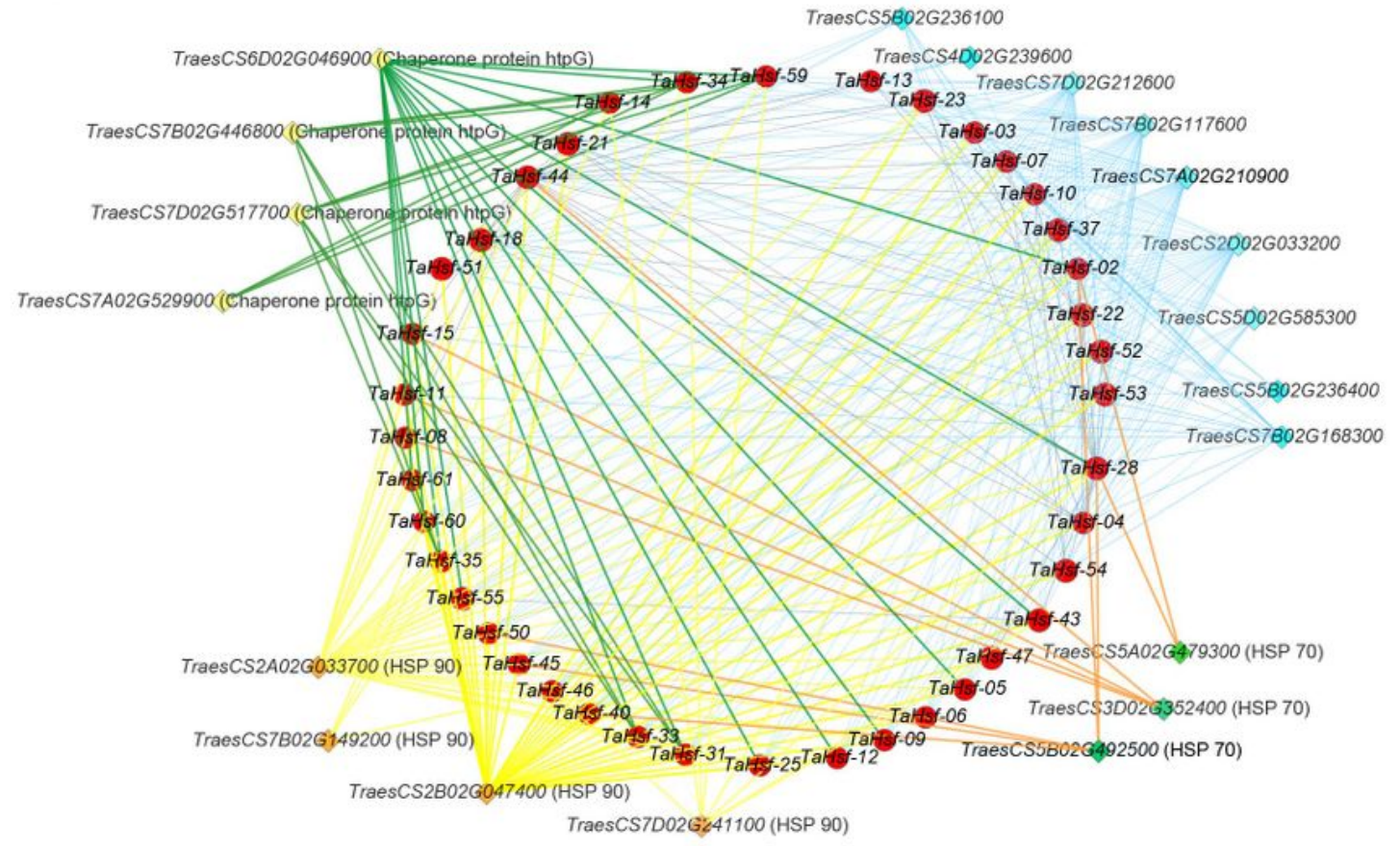

\section{Figure 7}

Interaction network between TaHsfs and related genes. a. Interactions between TaHsfs where the arrows denote regulation. b. Interactions between TaHsfs and other genes. The red circles represent TaHsfs and the hexagons in other colors represent the genes that interact with them. Lines connecting two genes show that an interaction exists between them. 


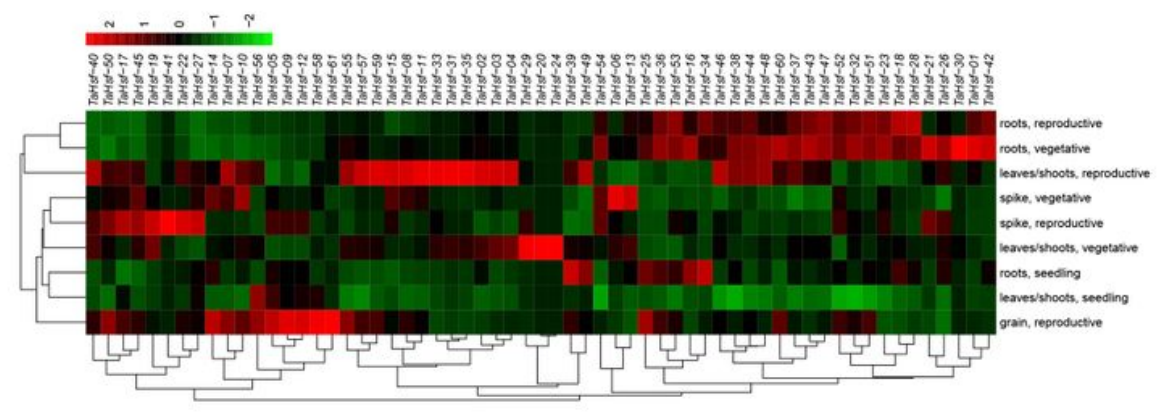

$8 \mathrm{~B}$
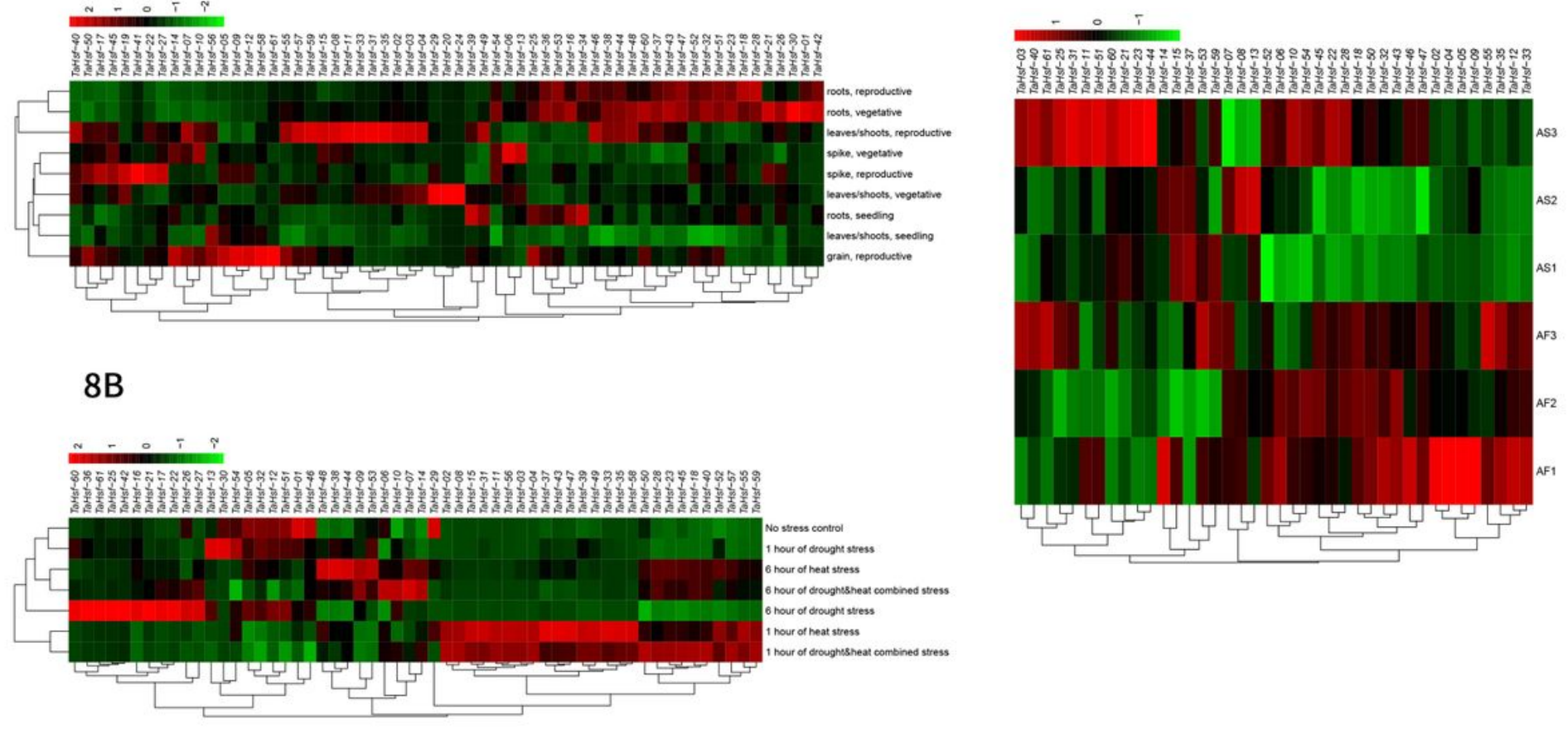

Figure 8

Expression profiles for TaHsfs in different condition. a. Heat map of TaHsfs in nine different tissues and growth stages under normal conditions. b. Heat map of TaHsfs under five different abiotic stress conditions and in a normal control. c. Heat map of TaHsfs in the thermosensitive male sterile wheat KTM3315A at two different temperatures. Heatmap obtained using the count values from RNA-seq analysis.

\section{Supplementary Files}

This is a list of supplementary files associated with this preprint. Click to download.

- supplement1.xlsx

- supplement2.xIsx

- supplement2.xIsx

- supplement4.xlsx

- supplement5.xIsx

- supplement5.xlsx

- supplement7.pdf

- supplement8.pdf

- supplement8.pdf

- supplement10.docx 